\title{
Review Techniques and Fundamentals of Internet Access Solutions: MANET- INTERNET Integration Scenario
}

\author{
Udai Shankar \\ IT Department \\ IIMT Engg. College \\ Meerut, India
}

\author{
Rakesh Kumar \\ CSE Department \\ MMM Engg.College \\ Gorakhpur, India
}

\begin{abstract}
Mobile Ad-hoc networks (MANETs) are formed by the association of the mobile devices, usually capable of multihop communication among themselves even if there is no networking infrastructure and central administration to control its operation. Need for the ubiquitous computing lead to connect the MANETs to the fixed IP Network i.e. internet. To connect the MANET to the internet first it might discover internet gateway through which it can communicate. The discovered gateway may be the mobile node within the MANET or it may be any fixed external node outside the MANET. The gateway works as the default router for all the nodes of the MANET through which all the incoming/outgoing packets are routed between the MANET and the internet. Discovery of the efficient internet gateway is very challenging task. Although So many solutions are proposed and implemented by the different authors/researchers but still no unique and robust solution is discovered so far, therefore a deep investigation and evaluation of the existing proposal is required to investigate the robust \& flexible solution. This paper discusses the fundamentals of MANET-INTERNET integration and address systematic evaluation of the current proposals with issues \& challenges remains unresolved. The aim of this paper is to build the strong foundation to discover an efficient, robust and flexible internet access solution that has not been discovered so far. This review paper concludes with further points of investigation
\end{abstract}

KEYWORDS: MANET, AODV, TTL FA, Gateway Discovery, Internet, Routing Protocols.

\section{INTRODUCTION}

A MANET is an autonomous collection of mobile users that move arbitrarily and communicate over multi-hop relays. The topology of the network may change unpredictably and dynamically. There is no pre-established infrastructure or backbone and centralized administration in the MANET. The MANETs are generally envisioned to operate as stand-alone networks, which mean that the traffic will be restricted within the MANET. All members in the MANET equally participate in the routing information distribution and route maintenance, by running the same routing protocol. Efficient ad hoc routing protocols must be adaptive to topological changes and traffic demands. MANETs can be deployed for temporary communications, such as conferences, emergency rescue, disaster relief and military missions [1].

When connecting MANETs with the Internet, the Routing Interoperability becomes a crucial challenge. Ad hoc routing protocols are originally designed for stand-alone MANETs where no intervention of any centralized router exists. In contrast to the Internet Protocol (IP), under a pure ad hoc routing protocol, every node must perform as a router and participate in route discovery and maintenance for other nodes. The ad hoc nodes cannot obtain the routing information beyond the scope of the MANET. When ad hoc routing is considered within the realms of IP-based networking, an ad hoc routing protocol is not capable of handling the communications across the boundary between MANET and the Internet. Therefore, the interoperability (or interfacing) between IP routing and ad hoc routing requires attention. Other challenges also emerge related to IP mobility issues in the Internet-integrated MANET. [2]. If any node within the MANET wants to access internet applications then it should be integrated with the internet. To establish the connection between the nodes within the MANET and INTERNET, a gateway is required to be discovered to route the information between the MANET nodes and Internet. There are different approaches for internet gateway discovery, such as proactive, reactive and hybrid. Proactive approaches are table driven, reactive approaches are source driven and hybrid approach is the combination of both of these [3]. Internet gateway may be a mobile node within the MANET (fig 1a) or it may be any external fixed node outside the MANET (fig 1b).

Different Protocol architecture is required for connectivity of MANET with Internet. TCP/IP suite and MANET Protocol Architecture use different languages. The Mobile devices in MANET share limited storage and less computational capabilities. They heavily depend on other hosts and resources for data access and information processing [4]. A Gateway, that must be able to translate between these "two languages", must understand both [5]. These networks are autonomous where a number of mobile nodes equipped with wireless interfaces

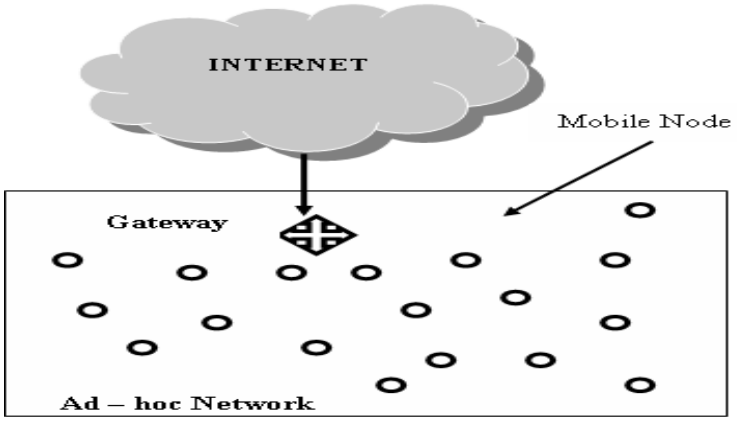

Fig. 1a: MANET-INTERNET Integration With Mobile Gateway 


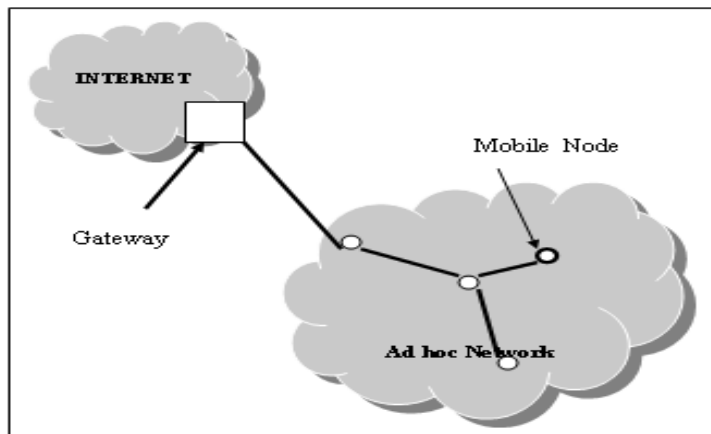

Fig. 1b: MANET-INTERNET Integration With Fixed Gateway

communicate with each other directly or through other nodes [6].In general, MANET topology is dynamic and asymmetrical; because of node departure and new node arrival during the connectivity time among the nodes, the nodes communicate over wireless links have a different transmission range [7].This paper discusses the fundamentals of MANETINTERNET integration and address systematic evaluation of the current proposals with issues \& challenges remains unresolved. The aim of this paper is to build the strong foundation to discover an efficient, robust and flexible internet access solution that has not been discovered so far. This paper concludes with further points of investigation.

The rest of the paper is structured as follows: Section $2 \& 3$ describes the Internet routing protocols and IP address auto-configuration respectively, while different issues and Challenges encountered in providing internet connectivity to the MANET and MANET-INTERNET integration protocol stack are discussed in section 4 and 5 respectively. Section 6 highlights the Internet Gateway discovery approaches and Care-of-Address provisioning for mobile nodes is explained in section 7. Review of existing internet access solution is presented in section 8 and section 9 presents the comparative study of the existing proposal then finally section 10 concludes the paper with further points of investigation.

\section{INTER-INTRA DOMAIN INTERNET ROUTING PROTOCOLS:}

Routing information or packets in Internet is IP address based. Each IP address consists of network id and host id portion. Routing decisions are taken on the part of routers for packets based on the network id portions of the destination IP addresses [6]. The IP addresses of nodes within the same network thus share the common network id whereas the node address portion of the IP address identifies a specific node in the network. The highest level of the Internet hierarchy consists of a number of Autonomous Systems (ASs). Each Autonomous System is a distinct routing domain. Routers communicate with each other within an Autonomous System using intra-domain routing protocols through core routers, which are also known as Interior Gateway Protocols. Border Gateway routers are used to interconnect different Autonomous Systems and communicate using inter-domain routing protocols among the distinct autonomous systems (ASs). Exterior Gateway Protocols are used to exchange routing information between Autonomous Systems and most commonly used routing protocol is BGP and now its version 4. As shown in fig, 2.

An older IGP is the Routing Information Protocol (RIP) [9]. RIP enables routers within an AS to exchange locally obtained information so that all routers within an AS have a coherent and up to date picture of how to reach any host within the AS. The principle functionality of RIP is that routers proactively advertise their routing tables to neighbor routers periodically. The hop count to a destination node is used as a metric for routing decisions. If a specific connection between two routers breaks the routers will use an alternative route for packet delivery.

Another IGP used is called Open Shortest Path First (OSPF) [10]. OSPF is a member of the "link state" family and commonly used nowadays. Instead of exchanging hop distances to destinations, routers maintain a map of the whole network that will be updated quickly if a change in the network topology is detected.

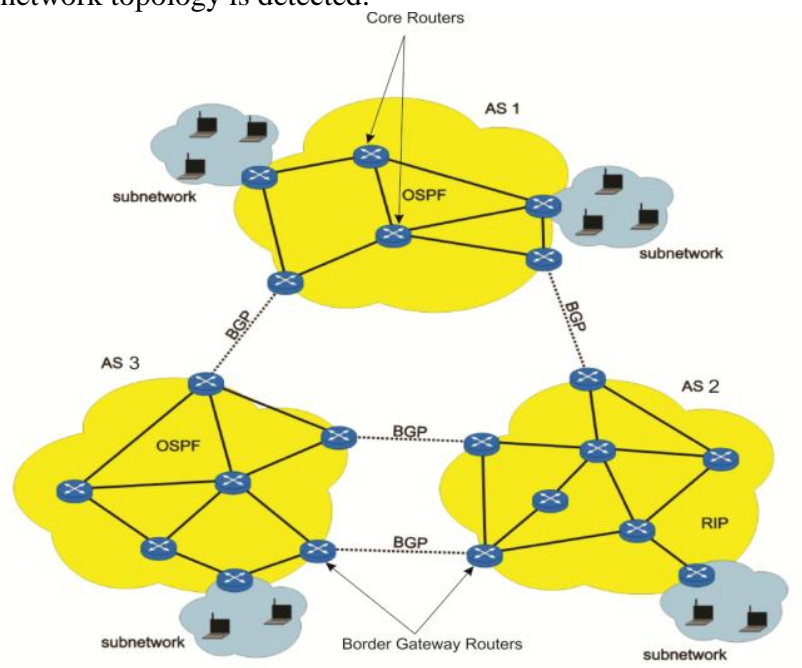

Fig. 2: Internet Architecture consisting autonomous systems

These maps (the link state database) is used to compute more suitable routes than RIP because OSPF uses more metrics like bandwidth, hop count, and reliability of a link. In OSPF a router is aware of all links between all routers of an AS.

On the other hand, one AS shares routing information with other ASs using the Border Gateway Protocol (BGP) [11]. BGP provides connectivity between all ASs and therefore, BGP is essential for the Internet functionality. BGP exchanges routing tables to other ASs on demand, i.e. when a change in the network topology was detected. For example a change occurs when a new AS is added to the Internet. Then this new AS announces itself to its neighbor ASs. The neighbor ASs gives their AS routing table to the new AS. As a result, every AS knows how to reach any other AS.

\section{IP ADDRESS AUTOCONFIGURATION:}

Addressing mechanism for the MANET nodes is flat addressing scheme and the node are assigned the IP addresses any subnet known as local address and is valid within that network only. When the mobile node has discovered the appropriate gateway, to exchange the packets between the mobile node and internet through the gateway the source node addresses prefixes should be the same as the gateway prefix. Ad Hoc node needs an address autoconfiguration mechanism in order to configure a global routable and topological correct address. in order to avoid other solutions like Network Address Translation (NAT) 
[12].several independent solutions have been proposed on interconnecting MANETs and the internet .most of the solutions are related to the issues of discovering internet gateways and auto-configuring global IP addresses that are routable within the internet. Usually auto-configuration of IP addresses in MANET is also required even when the MANET is isolated from external networks. Standalone or hybrid adhoc networks merged or partitioned by the process of network merger or partitioning process as a consequences of node mobility and/or wireless link environment, causing the duplicacy of the IP addresses that should be addressed by the duplicate address detection (DAD) mechanism. Autoconfigured addresses are likely to have life time associated with them, and after the life time expires use of the address should be immediately disconnected or negotiated. Address auto-configuration solutions should work well even when some nodes are temporarily disconnected or asleep. When duplicate addresses are detected, those nodes with conflicting addresses must resolve the conflict [13].

The IP address assignment for the nodes is performed using DHCP (Dynamic Host Configuration Protocol), it is better for IP address assignment in the fixed wired/or wireless nodes but not suitable for the MANETs nodes, as frequent problem is arises of duplicating of the address due to the merging and partitioning the networks. Dynamic address configuration was first implemented by the Dynamic Host Configuration Protocol (DHCP) [14].

\section{ISSUES AND CHALLANEG IN MANET- \\ INTERNET INTEGRATION:}

Currently there is a plethora of proposals to solve the problems of MANET-INTERNET integration. Many of the designs suffer from complexity and design solutions that have not been properly evaluated so far.

The main issue in MANET-INTERNET integration is the design of internet connectivity for MANETs that can handle node mobility both at micro and macro level that is within the same MANET domain and between the different MANET domains. having continuous and uninterrupted internet connections whenever there is at least one potential route to one or more gateways. Three challenging sub problems in MANET-INTERNET integration may be defined as-

i) Determining the node's location

ii) Discovering gateways and

iii) Establishing and maintaining consistent forwarding states to the gateways.

The natures of these problems are different depending on the assumptions for the specific scenario. Unless the scenario is very specific or there is an administrative entity in the network, it is hard to make any assumptions on what the network looks like. An ad hoc network is, by definition, to some degree unmanaged. Under those circumstances it is not possible to assume that there is, for example, only one gateway, that nodes move in a certain way or that nodes use a specific prefix for their configured IP address. Hence, it is argued that a general Internet connectivity solution must be robust enough to handle the most challenging scenarios. Such a scenario is defined with the following assumptions:

i. There might be multiple gateways to the Internet.

ii. Nodes are mobile, at both micro and macro scales.

iii. The routing protocol is reactive and hop-by-hop, i.e., each node has a limited horizon in the view of the network and only knows the next hop towards a destination.

iv. Nodes do not share a common IP-prefix [15].
Based on the above $\mathrm{i}$ to iv challenging scenario assumptions, their implications on the sub problems i to iii can be described as below:

Multiple Gateways: Since every node is a potential router and there is no sole administrator, a node might also be a gateway. Any node with an Internet connection could potentially offer that service to other nodes in the ad hoc network if it so wishes. Multiple gateways have implications for problem ii) in that discovering several gateways gives the option to either select one gateway at a time or use several at once. For iii) in that a TCP connection might break if the forwarding state is suddenly re-pointed to another gateway somewhere along a path without the explicit knowledge of the source of the connection.

Mobility: For the second point it is argued that nodes might be (micro) mobile within a MANET, but they should also be able to seamlessly move between different MANETs and be (macro) mobile between a MANET and the Internet. The latter assumption might require, e.g., Mobile IP and hence integration with the Internet connectivity system. The mobility assumption also has implications on i) and iii). Agent registration must match that of the currently used gateway and if a route switches to another gateway, the source nodes using that route must be notified so that they can reregister with the new agent there.

Routing: The mobility assumption implies a routing protocol that reacts swiftly to topology changes. The implications of reactiveness for problem are that the protocol only maintains a partial network state (routes to active destinations only). Therefore, in combination with prefix less addressing, there is no way to easily determine node locations, i.e., whether a node is located in the MANET or in the Internet. For ii) it is important that the Internet connectivity design supports reactive gateway discovery. The partial network view of the routing protocol in combination with hop-by-hop forwarding is a problem for iii). Each hop on the forwarding path runs the risk of repeating the problem of determining node locations for every packet.

Addressing: Prefix-less or a flat address are a common assumption in the ad hoc network research community and is a requirement for macro mobility. A node should, in line with the Mobile IP specification, be able to bring its preconfigured home address into the ad hoc network and use it for routing. Hence, there is no common prefix among nodes and the ad hoc network is flat in both a routing and addressing sense. As mentioned above, this has implications for problem i) in combination with reactive routing. Using a proactive protocol or prefixes/subnets solve the problem since node locations can be determined either by checking the routing table or by examining the IP address prefix of the destination address. In addition to the functionality for operation in the worst case scenario, an Internet connectivity design could offer optional functionality for flexibility, for example, exploiting multiple gateways for the purpose of multi-homing or load balancing $[15,16]$.

Other key challenges are the design challenges that are as ad hoc routing protocols are originally designed for standalone MANETs where no intervention of any centralized router exists. In contrast to the Internet Protocol (IP), under a pure ad hoc routing protocol, every node must perform as a router and participate in route discovery and maintenance for other nodes. The ad hoc nodes cannot obtain the routing information beyond the scope of the MANET. When ad hoc routing is considered within the realms of IP-based networking, an ad hoc routing protocol is not capable of handling the communications across the boundary between MANET and the Internet. Therefore, the interoperability (or 
interfacing) between IP routing and ad hoc routing requires attention to maintain connectivity globally when moving from one network to another. To support the above challenges MANET-INTERNET integration must support the IP mobility as well [1]

\section{MANET-INTERNET INTEGRATION PROTOCOL STACK:}

Comparison of the basic protocol stack for mobile ad hoc network with OSI model and TCP/IP suite, presents a better understanding of the differences in their protocol architecture. Figure 3 shows these protocol stacks $[12,6]$.

OSI model provides a layered framework for communication between networked computers. TCP/IP suite was designed before the OSI model. The lower four layers are same as the OSI model. The top most layers, i.e. the application layer is a combination of the application, presentation and session layer of the OSI model. The MANET protocol stack is similar to the TCP/IP suite. Only the network layer differs for these two protocol stacks. In case of MANET OSI MODEL

\begin{tabular}{|c|}
\hline APPLICATION \\
\hline PRESENTATION \\
\hline SESSION \\
\hline TRANSPORT \\
\hline NETWORK \\
\hline DATA LINK \\
\hline PHYSICAL \\
\hline
\end{tabular}

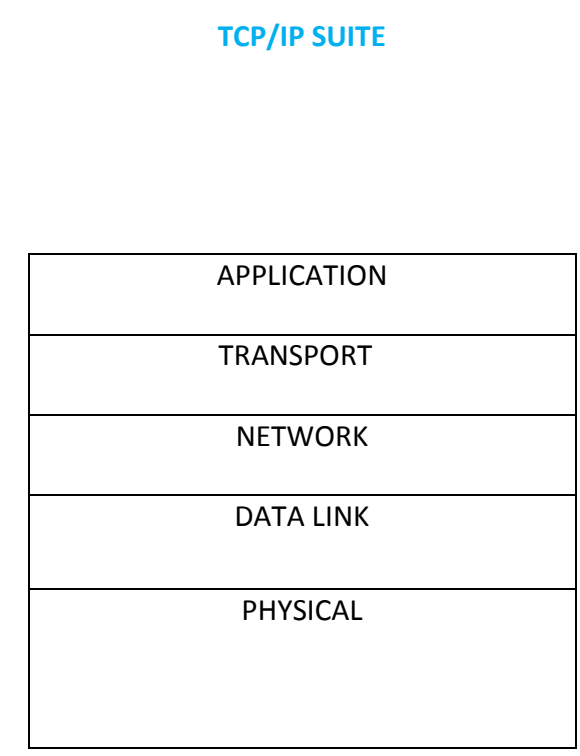

Fig. 3: Basic Protocol Stack of Network Routing

MANET NODE

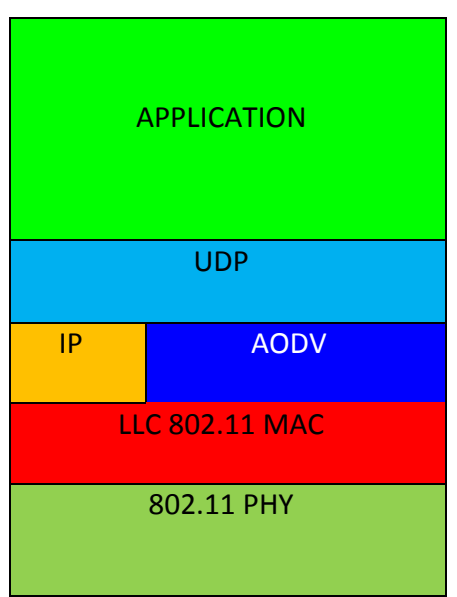

GATEWAY

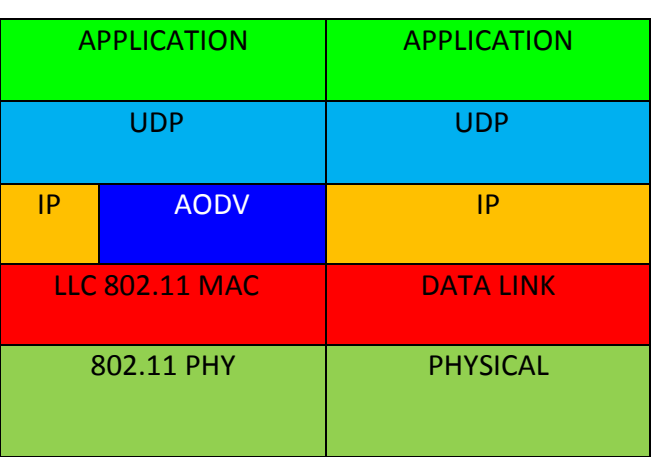

protocol stack, this layer is divided into two parts - network and ad hoc routing.

Standard Internet routing protocols are used in the network part. MANET nodes use an ad hoc routing protocol for handling the routing within the ad hoc network. Mobile nodes run protocols that have been designed for the wireless channels and are capable of decentralized direct mode operation in the physical and data link layers.

Figure 4 shows the protocol architecture needed for interconnection between the MANET and the Internet. The Internet nodes use the TCP/IP suite and the MANET nodes use the MANET protocol stack discussed above. Whenever a mobile node wants to send a data packet to the Internet, it has to forward it to the gateway. The gateway then transmits the packet to the corresponding node in the Internet. Thus the gateway functions as a bridge between the MANET and the Internet. It has to translate between these two different protocols and must understand both. Therefore, it needs to implement both the MANET protocol stack and the TCP/IP suite.

MANET PROTOCOL STACK

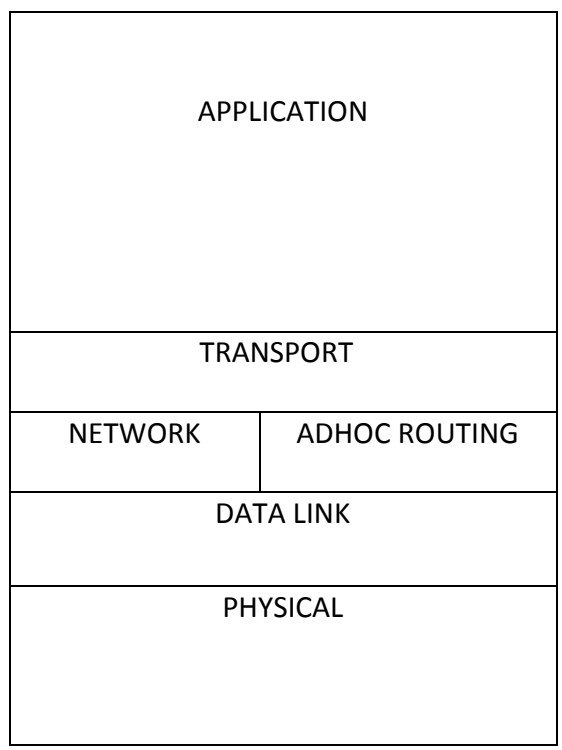

MOBILE HOST

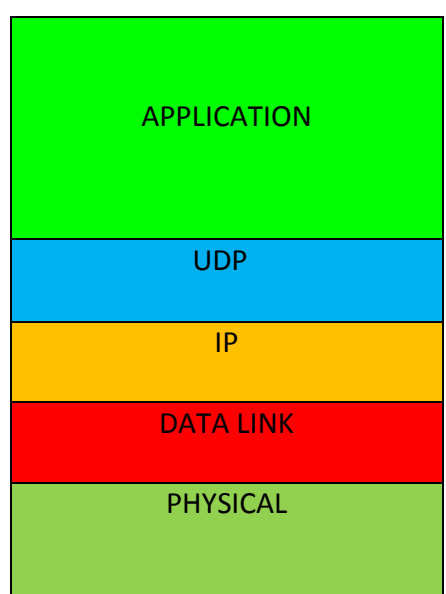

Fig. 4: Gateway Protocol Stack: MANET-INTERNET Integration 


\section{INTERNET GATEWAY DISCOVERY APPROACHES:}

For access to global services, an Internet Gateway (IGW) in the access network can provide Internet connectivity for nodes in the MANETs. Mobile nodes from Ad Hoc network can use this route to send/receive packets addressed to or from Internet. The standard Ad Hoc routing protocols do not provide the functionality of detecting Internet gateways, thus the protocols have to be extended. The extensions to the standard Ad Hoc routing protocols are based upon special Ad Hoc routing messages. Depending on who initiates the gateway discovery, these approaches can broadly be classified into the following three categories $[12,6,17]$

\section{A. Reactive gateway discovery. \\ B. Proactive gateway discovery. \\ C. Hybrid gateway discovery.}

Ad-Hoc on Demand Distance Vector Routing (AODV) protocol has been studied to provide the connectivity to the wired network (internet and other resources) via gateway. (A modification in the source code of AODV in NS 2 in accordance with the Internet draft "Global connectivity for IPv6 Mobile Ad-Hoc Networks" which presents a solution where AODV is used to provide Internet access to mobile nodes [18]. To always keep the mobile nodes updated about the information of the gateways three mechanisms have been proposed to transmit the information about the gateways to the mobile nodes. The configuration phase between the Fixed network (Gateway) and mobile nodes in the mobile Ad-Hoc network can be initiated by the Fixed network(Gateway), by the mobile node or a mixed approach can be used to configure the mobile nodes and the fixed network. These gateways discovery mechanism are discussed as below:

\section{A. Reactive Gateway Discovery}

The reactive gateway discovery is initiated by a mobile node that is to initialize or update information about the gateway. The mobile node broadcasts a RREQ_I to the ALL_MANET_GW_MULTICAST address, i.e. the IP address for the group of all gateways in a mobile Ad-Hoc network. Thus, only the gateways are addressed by this message and only they process it. Intermediate mobile nodes that receive the message just forward it by broadcasting it again. Since the message format is RREQ, which has a RREQ ID field as discussed in Section V.A, duplicated RREQ_Is are discarded. Upon receipt of a RREQ_I, a gateway unicasts back a RREP_I which, among other things, contains the IP address of the gateway. The advantage of this approach is that RREQ_Is are sent only when a mobile node needs the information about reachable gateways. Hence, periodic flooding of the complete mobile Ad-Hoc network, which has obvious disadvantages. The disadvantage of reactive gateway discovery is that the load on forwarding mobile nodes, especially on those close to a gateway, is increased.

\section{B. Proactive Gateway Discovery}

The proactive gateway discovery is initiated by the gateway itself. The gateway periodically broadcasts a gateway advertisement (GWADV) message which is transmitted after expiration of the gateway's timer, ADVERTISEMENT_INTERVAL. The time between two consecutive advertisements must be chosen with care so that the network is not flooded unnecessarily. All mobile nodes residing in the gateway's transmission range receive the advertisement. Upon receipt of the advertisement, the mobile nodes that do not have a route to the gateway create a route entry for it in their routing tables. Mobile nodes that already have a route to the gateway update their route entry for the gateway. Next, the advertisement is forwarded by the mobile nodes to other mobile nodes residing in their transmission range. To assure that all mobile nodes within the mobile AdHoc network receive the advertisement, the number of retransmissions is determined by NET_DIAMETER defined by AODV. However, this will lead to enormously many unnecessary duplicated advertisements. A conceivable solution to the problem that occurs due to these duplicated advertisements. Although the problem of duplicated broadcast messages can be solved, one disadvantage remains. This disadvantage, which is general for all proactive approaches, is the fact that the message is flooded through the whole mobile Ad-Hoc network periodically. This is very costly operation. Limited resources in a mobile Ad-Hoc network, such as power and bandwidth, will be used a lot.

\section{Hybrid Gateway Discovery}

In the hybrid gateway discovery approach the gateway periodically broadcasts the GWADV message. The TTL is set to ADVERTISEMENT_ZONE so that the advertisement message can be forwarded only up to this maximal number of hops through the ad hoc network. The mobile nodes within this region receive this message and act according to the proactive approach. The nodes outside this region discover the default routes to the gateways using the reactive approach.

\section{CARE-OF-ADDRESS PROVISIONING FOR MOBILE NODES:}

Mobile nodes running Mobile IP can acquire care-of addresses in two ways. In the first method, a foreign agent (FA) must be available on the foreign network. This agent advertises one or more care-of addresses in its Agent Advertisements. Mobile nodes can then use one of those careof addresses to obtain Internet connectivity fig 5 . The other method for a mobile node to obtain Internet connectivity is to acquire a co-located care-of address. This type of care-of address is used when a foreign agent is not available on the network. At minimum, a gateway between the wired and wireless networks must be configured to advertise network prefixes that are routable on the given network. When such a gateway is available, mobile nodes can use the advertised prefix to configure their own care-of addresses. To obtain a unique care-of address, a mobile node must select a unique identifier to append to the advertised network prefix. A mobile node does not necessarily know the care-of addresses of the other mobile nodes within the wireless network. Hence, it must perform duplicate address detection to ensure that its selected address is unique. The following method is based on that described in [19].

When a node requires a unique IP address, it first selects a random host ID from the range 2048- $\left(2^{(32-1)}-1\right)$, where $n$ is the number of significant bits in the advertised network prefix. The node then appends that host ID to the prefix advertised by the Internet gateway. This is the IP address for which it performs duplicate address detection. The node then selects a random, temporary host ID in the range 02047 and appends this value to the advertised network prefix. This ID serves as a source IP address for the short period while the node performs duplicate address detection.

The node creates an Address Request (AREQ) by placing its randomly selected source IP address, as well as its temporary IP address, in the AREQ and broadcasts this request to its neighbors. When a mobile node receives an 
AREQ message, it creates a reverse route entry for the node indicated by the temporary IP address in the AREQ. The node then checks whether its own IP address matches the requested address in the AREQ. If the node's IP address does not match the requested address, it rebroadcasts the packet to its neighbors.

On the other hand, if the node has the same IP address as that requested by the AREQ, then the source node is requesting an IP address that is already in use. In this case, the node with the duplicate IP address creates an Address Reply (AREP) packet. It places the requested IP address in this message, and unicasts this packet to the node that requested the address. The reverse route that was created by the AREQ broadcast is used to route the AREP back to the source node.

When a node originates an AREQ, it sets a timer to wait for the reception of an AREP message. If no AREP is returned for the selected address within a timeout period, the node retries the AREQ up to some maximum number of times. If, after all retries, no AREP is received, the node assumes that the address is not already in use, and that the address can safely be taken for its own. On the other hand, if the node does receive an AREP within the discovery period, and if the requested IP Address included in the AREP matches the address it was requesting, then this indicates that another node within the ad hoc network is currently using that IP address. In this case, the node randomly picks another host ID from the same 2048-( $\left(2^{(32-1)}-1\right)$ range, and begins the duplicate address detection again [22].

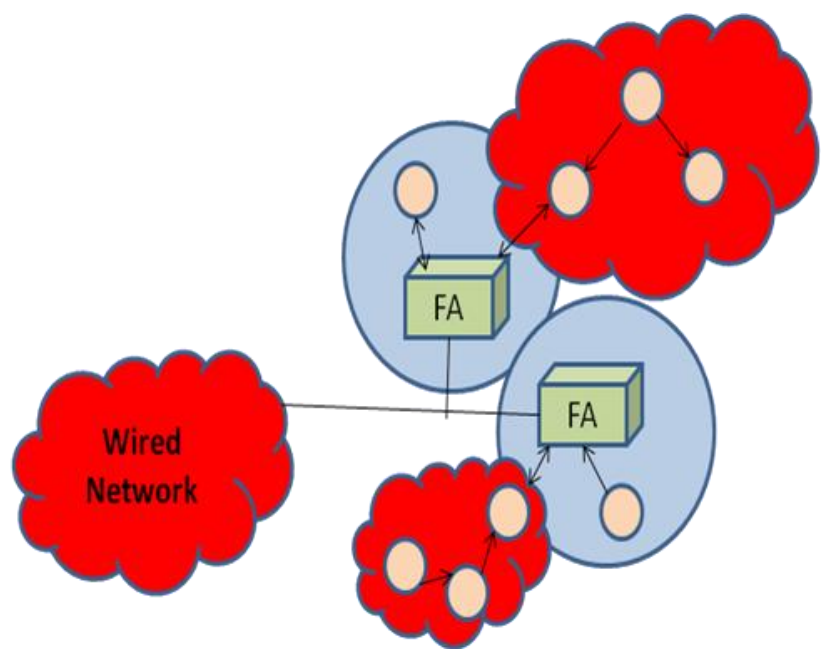

Fig. 5: Ad hoc Network with internet connectivity

\section{REVIEW OF EXISTING INTERNET ACCESS SOLUTIONS}

To access the internet application through MANET nodes, it needs the access point to provide bridge between MANET and internet called gateway. It requires mapping the flat addresses of the ad mobile node to the global topological address to communicate with the host located in the IP network. It is also paramount requirement of the mechanism to deal with the mobility of the visiting node, especially when node moves from one network to the other networks. Due to leaving and joining the networks the problem of duplicate addresses encounters most frequently for the reason of network partitioning and merging. For this reason efficient scheme is required to duplicate address detection, its resolution and care-of-address visiting node. Several solutions have been proposed to deal with the integration of MANETs to the Internet. Most of the proposed solutions require the addition of gateways and differ in the design and functionality of the gateways, number of occurrences, and the routing protocols used within the Ad Hoc network. Since Internet gateways have two interfaces they are part of the Internet and the Ad Hoc network simultaneously. They understand the Internet protocol (IP) as well as a MANET routing protocol (e.g. AODV). Mostly, the existing approaches consider only static gateways to connect MANET nodes to the wired Internet and very few have considered dynamic approaches. This paper briefly discusses solutions for both static Internet gateways and dynamic Internet gateways [19, 20].

\section{Static gateway discovery solutions:}

Royer E.M. [18], Proposed Mobile IP which was supported by IPv4 ad hoc networks with AODV routing protocol. The proposed scheme has a proactive agent solicitation procedure with AODV route search to register to Mobile IP. It distinguishes the location of destination nodes using FRREP of FA, when a packet is sent to the Internet. In addition, it is capable of packet routing using default routing of FA. However, this proposal does not consider the selection between multiple FAs. Also, it delays the connection setup time because this proposal first need to conclude that the destination is not within the ad hoc network before a mobile node can use the FA.

To get the best of reactive and proactive approaches, hybrid schemes may be used. Ratanchandani et al. [21], describe a hybrid solution within the context of Mobile IP. Foreign agents (FA) proactively send advertisements to their closest nodes, while farthest ones operate on demand. To control the scope of the advertised messages the Time to Live (TTL) field of the IP header is set to a fixed value. The problem is that there is not a best TTL for a range of scenarios and network conditions.

Shin [22], proposed NAT-based MANETINTERNET integration access. Periodic gateway discovery mechanism is used based on periodic HELLO message. HELLO scheme is extended as e-HELLO to contain the RGS (Reachable Gateway Sets) information over the ad hoc networks to inform its neighbor node when HELLO message is broadcasted and updates route entries for the gateways. NAT-based table for source node and proxy RREP (P-RREP) scheme is used to maintain the connectivity with the gateway. Mobile node can change its current gateway only when current session is over when node moves away the range of the default gateway.

Ruiz. P.M [23], the authors propose an adaptive gateway discovery mechanism based on the hybrid discovery approach that modifies the scope of the GWADV (Gateway Advertisement) messages sent by the gateways to obtain the maximal benefit in terms of overhead savings by avoiding sources to flood the network asking for gateways. The same authors propose in [24] an adaptive gateway discovery mechanism based on the hybrid discovery approach that modifies the scope of the GWADV messages sent by the gateways to reach the maximal number of active sources. A comparison between these gateway discovery schemes with already existing ones is done in [25], where both adaptive approaches have been evaluated with similar results and it has been demonstrated that they outperform existing schemes.

Khaleel [26], described extension of approach for gateway selection scheme. The path is updated to the gateway on the request of mobile node which facilitates handoff from one gateway to another gateway. This scheme also maintains continuous connectivity to the fixed host. Another extension is that routing queue length and minimum hop count metric is 
not only used to discover the routes to the gateway but also for routing in the local ad hoc domain among ad hoc host. The occupancy level of each of node is updated after a short interval of time. This reduces the delay and increase packet delivery ratio.

Bin et al. [27], proposed an adaptive gateway discovery scheme that can dynamically adjust the TTL value of Agent Advertisements (GWADV messages) according to the mobile nodes MANET Internet traffic and their related position from Internet Gateways with which they registered. This protocol provides Internet access to MANET mobile nodes using mobile IP.

Hamidian [28], the authors extended the AODV routing protocol to the Internet to achieve the interconnection between the MANET and the Internet. The paper discusses and evaluates three approaches for gateway discovery. The authors implement these three schemes in network simulator 2 (ns-2) and compare them by means of simulation study. They also discuss the advantages and disadvantages of the three approaches. The gateway discovery method follows a similar approach based on. However, those approaches have a fixed TTL for proactive gateway advertisements and do not reflect dynamic network conditions.

R. Wakikawa [29], the authors proposed an approach to global Internet connection over the IPv6 MANET environment, where mobile nodes in the ad hoc network are configured with new globally routable IP addresses based on the neighbor discovery protocol (NDP) of IPv6 or route searching procedure of on-demand routing protocol. This paper defines two different mechanisms to discover Internet gateways: periodic flooding of gateway advertisement (GWADV) messages from the gateways and reactive flooding a gateway solicitation (GWSOL) message from nodes. The periodic flooding using GWADV is completely proactive, whereas the reactive flooding using GWSOL is completely reactive. GWADV and GWSOL messages can be implemented by simply adding an "I" flag to existing route request (RREQ) and route reply (RREP) messages. However, this proposal does not give any metric to select a gateway.

The optimization of the timing of MRA messages was studied in R.Kumar [30]. In this study, the authors suggest that the appropriateness of broadcasting a MRA message depends on the number of active sources that communicate to external hosts as well as the number of intermediate nodes that forward the packets to the internet gateway. With these two parameters, the so-called Regulated Mobility Degree (RMD) is defined. When this factor overpasses a pre-established threshold, the MRA message is sent.

Trujillo [31, 32], proposed process optimized adaptive gateway discovery approach to provide internet connectivity in multi-hop ad hoc networks. Modified router advertisement (MRA) message scheme is used to discover the gateway. Claims that mechanism can be implemented using either scheme, reactive, proactive or hybrid. Time interval $\mathrm{T}$ is defined for MRA advertisement as beacon interval. Control system function is defined to adjust that is proportional to MRA massages received from the gateway neighbors and also real mobile node close to the gateway. It is also defined that the probability $p$ that a node is near a gateway, regardless of the model chosen for mobility can be given by binomial distribution.

Carman [33], proposed hybrid gateway discovery scheme with modified AODV routing protocol for routing in ad hoc domain. Scheme differentiates between the best-effort and real- time services. QoS are also discussed for real time services. Threshold is discussed for end-to-end delay to improve the QoS for real time services. QoS model named DS-SWAN is proposed to improve the real time flows. QoS _LOST parameter is defined to assume packet lost is end-toend delay cross the threshold. Ratio between the number of real time sources having latency problem and total number of real time sources using gateways and a threshold parameter is also discussed. Destination keeps track of the lost messages and inform to real time source traffic. Also suggest that to reduce the end-to-end delay of the lost message the message will be forwarded as broadcast packet.

Ruiz et al. describe in [34], an adaptive algorithm which selects the TTL of the gateway advertisements according to the number of hops between the traffic sources and the gateways. This approach tries to limit the huge overhead which is provoked by the reactive scheme when there are many traffic sources in the network. At the same time, the overhead of the proactive algorithm when the number of gateways increases is also reduced. The same paper performed an analytical study where it is shown how the reactive gateway discovery has a big impact on the overall performance when there are many traffic sources.

R. Kumar [3], discussed about minimum hop metric. A Mobile node uses minimum hops to communicate to a fixed host using gateway. Sometimes, a minimum hop path is not sufficient if there are some waiting packets in the queue. The Authors analyzed existing load aware routing protocols in MANET and devise a proactive load aware routing scheme. This scheme uses the interface queue occupancy and min hop metrics. The Handoff from one gateway to other gateway is also discussed. When a mobile node receives gateway advertisements based on interface queue interface queue and minimum hop, the node updates its default route to the gateway.

Geetha [35], described the Protocols used in Mobile Ad hoc Network. The gateway is used to communicate MANET nodes with Internet. The Authors surveyed the AODV and DSDV protocols. The Protocols are analyzed and Performance of AODV is better as compared to DSDV Protocol.

Zhuang [36], proposed adaptive algorithm for MANET-INTERNET integration. Scheme uses the usual approach to route discovery and gateway discovery with new approach to remove the unidirectional links to avoid unnecessary control overheads. Each node in MANET maintain a neighbor node list (NNL) by sending HELLO messages to record its set of neighbors, with information about the node like neighbor IP address and its life time. RREQ (RREQ-I) is broadcasted along with this information. Nodes receiving the RREQ check for its presence in received RREQ, it it is there then between this node and broadcasting node link is bi-directional otherwise RREQ is discarded and it is not broadcasted further. Same scheme is adopted for gateway advertisement with additional information attached with GWADV message. Scheme adjusts broadcast range, GWADV interval and TTL value.

Majumdar [6], analyzed that users require huge amount of resources and services from the internet and for increasing the coverage area of MANET. So there is a need for integration ad hoc networks to the internet. The gateways are used which acts as bridges between these two protocol architectures. The gateway discovery scheme in hybrid network is a complicated task. The complexity increases due to greater number of sources. So AODV reactive routing protocol is extended to provide communication between MANET and Internet. The number of received packets increases with more number of sources, so traffic increases. The number of sources and number of gateway discoveries 
results the increase in traffic. These further results in higher routing load. The hybrid approach is a combination of proactive and reactive approaches, the routing load lies between these protocols.

Shahid [37], proposed the hybrid scheme for gateway discovery and selection based on on-demand gateway advertisement. Interface queue length and the total number of neighbors along a route are also considered in addition to the hop count to bypass the loaded and dense route to the gateway in order to reduce the delay and packet loss. It is proposed in discovery scheme that the mobile node want to internet access should first search its routing table for the available gateway and if available then set default route to the gateway, otherwise start solicitation process by broadcasting the GWDSC message with initial TTL value. Gateway advertisement process is initiated only when the gateway is triggered by the GWDSC message to reduce the routing overhead. The proposal states that the intermediate nodes to update two additional field interface queue length and total number of neighbors of the nodes along a route from a gateway while forwarding the message to the next nodes.

Erik [16], has designed the robust and flexible MANET-INTERNET integration approach using AODV routing protocol. Indirection approach is integrated using tunnels with the AODV routing protocol. Default route forwarding is also integrated to compare the two approaches. Similar gateway discovery and route setup mechanism is used for both default route and tunneling. Proxy RREP solution is used. The scheme use RREQ to determine the route in the MANET as normal process. A gateway replies by RREP to determine locality of destination. The address locality check at gateway is implemented through a prefix check or using a visitor list. Flag $G$ is used to mark gateways to indicate backup tunnels for faster hand off and I flag to distinguish internet host entry from normal MANET route entry. Internet

routing protocol. The mobile router sets up tunnels to every mobile node for which it is serving as gateway, and another tunnel to the HA using second interface.

Khan et al. [40], proposed a new approach for integrating MANET with the Internet by devising a protocol named Efficient DSDV (Eff-DSDV). The proposed framework uses one of the Ad Hoc mobile nodes as a Mobile Internet Gateway (MIG), which acts as a bridge between the connectivity scheme has been implemented into the AODV UU implementation. These code runes in both the ns-2 simulator and Linux with IPv4.

\section{Dynamic gateway discovery solutions:}

In [38], Ammari et al. proposed a mobile gateway based on three-layer approach using both Mobile IP protocol and DSDV Ad Hoc routing protocol. The first layer contains Mobile IP foreign agents; the second layer includes mobile gateways and mobile Internet nodes, which are one-hop away from Mobile IP foreign agents; the third layer has all MANET nodes and visiting mobile Internet nodes that are at least onehop away from mobile gateways. The second layer is to provide Internet connectivity to MANET nodes and, thus to help establish interaction between MANET nodes and the Internet. Mobile gateways are powerful MANET nodes and are designed in a way to use both Mobile IP protocol when they communicate with the Internet. The DSDV protocol is used for routing within the MANET. The integration framework considers using some border MANET nodes to connect the rest of MANET nodes to the Internet. These MANET nodes are referred as mobile gateways. A mobile gateway selects a closest and/or a least loaded foreign agent based on the distance and the load criteria. MANET nodes select a closest and/or least loaded mobile gateway.

Kock [39], integration between cellular system (GPRS) and ad hoc networks is presented using Mobile IP. The basic idea in the integration is using mobile routers as a gateway between the HA and ad hoc mobile nodes. It is assumed that gateways (mobile routers) in the ad hoc network are multi-interfaced. One interface is connected to the cellular system and the other connected to the ad hoc network using the ad hoc

two networks. The MIG runs the Eff-DSDV protocol and takes care of the addressing mechanisms to ensure the transfer of packets between MANET and Internet. This strategy does not require the flooding of the gateway advertisements for registration of mobile nodes with MIG. Ad Hoc routing protocol EFFDSDV and Mobile IP coordinate with each other to provide the connectivity. Eff-DSDV follows the conventional DSDV; however it reduces the packet loss due to broken links.

\section{COMPARATIVE SUMMARY OF THE CURRENT PROPOSALS}

Table 1: Comparative Summary of the Current Proposals

\begin{tabular}{|l|c|l|c|c|l|}
\hline \multicolumn{1}{|c|}{$\begin{array}{c}\text { Scheme/ } \\
\text { Author(s) }\end{array}$} & $\begin{array}{c}\text { Ad-hoc } \\
\text { Domain } \\
\text { Routing } \\
\text { Protocol }\end{array}$ & $\begin{array}{c}\text { Gateway } \\
\text { Discovery } \\
\text { Scheme }\end{array}$ & $\begin{array}{c}\text { Mobile } \\
\text { IP Used }\end{array}$ & $\begin{array}{c}\text { Multiple } \\
\text { Gateway } \\
\text { Support }\end{array}$ & \multicolumn{1}{|c|}{ Significant Features of the Scheme } \\
\hline R. Kumar [3] & AODV & Proactive & YES & YES & Load aware gateway selection \\
\hline Erik [16] & generic & $\begin{array}{l}\text { Reactive } \\
\text { with proxy } \\
\text { RREP }\end{array}$ & YES & YES & Flexibility to work with any MANET routing protocol \\
\hline Royer E.M. [18] & AODV & Proactive & NO & NO & $\begin{array}{l}\text { Distinguishes the destination node location using FRREP } \\
\text { of FA }\end{array}$ \\
\hline $\begin{array}{l}\text { Ratanchandani } \text { et } \\
\text { al. } \text { [21] }\end{array}$ & AODV & Hybrid & YES & YES & Fixed TTL value to control advertised messages \\
\hline Shin et al. [22] & AODV & Proactive & NO & YES & Proxy- RREP maintains connectivity to Gateways. \\
\hline Ruiz. P.M [23] & AODV & Hybrid & NO & NO & Reduces control overheads \\
\hline Khaleel [26] & AODV & Proactive & YES & YES & Reduce delay and increase PDF \\
\hline
\end{tabular}




\begin{tabular}{|c|c|c|c|c|c|}
\hline Bin et al. [27] & AODV & Reactive & YES & YES & TTL adjustment according to network scenario \\
\hline Hamidian [28] & AODV & $\begin{array}{l}\text { Proactive, } \\
\text { Reactive, } \\
\text { Hybrid }\end{array}$ & $\mathrm{NO}$ & YES & $\begin{array}{l}\text { AODV is extended and performance of three gateway } \\
\text { discovery schemes is analyzed with ns-2 }\end{array}$ \\
\hline R. Wakikawa [29] & generic & $\begin{array}{l}\text { Proactive, } \\
\text { Reactive }\end{array}$ & IPv6 & YES & $\begin{array}{l}\text { Mobile node's global address is configured using neighbor } \\
\text { discovery protocol (NDP) }\end{array}$ \\
\hline R.Kumar [30] & AODV & Proactive & YES & YES & $\begin{array}{l}\text { Periodic and adaptive advertisement are combined that } \\
\text { improve PDF and reduces overhead messages }\end{array}$ \\
\hline Trujillo $[31,32]$ & AODV & Proactive & YES & YES & $\begin{array}{l}\text { Decrease control traffic without increasing packet lost rate } \\
\text { and delay time }\end{array}$ \\
\hline Carman [33] & $\begin{array}{l}\text { Modified } \\
\text { AODV }\end{array}$ & Hybrid & IPv6 & YES & QoS support for real time services \\
\hline Ruiz et al. [34] & AODV & Proactive & YES & YES & Reduces gateway message advertisement overheads \\
\hline Zhuang [36] & AODV & Proactive & YES & YES & $\begin{array}{l}\text { Reduces control overheads by removing unidirectional } \\
\text { links }\end{array}$ \\
\hline Shahid [37] & AODV & Hybrid & YES & YES & $\begin{array}{l}\text { Bypass loaded and dense route to gateway to reduce delay } \\
\text { and packet loss }\end{array}$ \\
\hline Ammari et al. 38] & DSDV & Reactive & YES & YES & Load and distance aware selection of the foreign agent \\
\hline Kock [39] & DSDV & Reactive & YES & YES & $\begin{array}{l}\text { Support mobile gateway and provide interface between } \\
\text { GPRS \& Ad-hoc Networks }\end{array}$ \\
\hline Khan et al. [40] & $\begin{array}{l}\text { Eff- } \\
\text { DSDV }\end{array}$ & Proactive & YES & YES & $\begin{array}{l}\text { Does not require flooding of gateway advertisement for } \\
\text { registration of mobile node with mobile internet gateway }\end{array}$ \\
\hline
\end{tabular}

\section{CONCLUSION AND FUTURE DIRECTIONS}

This paper has presented a comprehensive review of solutions for integration MANETs with Internet. Most of the solutions proposed are based on Mobile IP mobility protocols. Solutions are investigated systematically and their limitations have also been dealt with. Maximum solutions presented are based on the fixed gateways, only very few solution proposed are based on mobile gateways. We have also presented fundamentals of MANET-INTERNET integration for better understanding of the solutions like, different connectivity issues $\&$ challenges, care-of-address for the mobile nodes at micro and macro level mobility.

This paper conclude that although much exercise has been extended by the different authors in this area and the solution provided are network scenario based and no unique and standard solution is discovered so far. Hence a deep investigation is required to be exercised to provide network scenario independent, robust \& flexible solution that can be operated independently of routing protocols and other network conditions.

Hence there is the future scope to work in the areas like, optimization of the control overheads occurred due to the nodes solicitations and gateways advertisements, selection of the optimal gateway from the multiple gateways available, multi-homing or load balancing means maintaining consistent forwarding states with the multiple gateways simultaneously, QoS provisioning between the MANET nodes and Internet host, extension of the IP based networks to include dead zones and cover long area and decrease in access points (APs), seamless roaming and efficient indirection towards the gateways.

\section{REFERENCES}

[1] Shuo Ding, "A survey on integrating MANETs with the Internet: Challenges and designs", Elsevier Journal of Computer Communications- 2008. [

[2] A. Safwat, H. Mouftah," "4G network technologies for mobile telecommunications', IEEE Network 19 (5) (2005) 3-4.

[3] Kumar Rakesh,Anil Kumar Surje and Manoj Mishra, "A Proactive Load-Aware Gateway Discovery in Ad Hoc Networks for Internet Connectivity", International Journal of Computer Networks \& Communications (IJCNC) Vol.2, No.5, September 2010

[4] Characteristics of Manet,"www.Saching.com/ Article/MANETMobile Adhoc NETwork /334"

[5] Kamaljit I. Lakhtaria and Prof. Bhaskar N. Patel, "Comparing Different Gateway Discovery Mechanism for Connectivity of Internet and MANET", International Journal of Wireless Communication and Simulation, Vol.2 No.1, 209, PP: 51-63.

[6] Koushik Majumder, Dr. Sudhabindu Ray, Prof. Subir Kar Sarkar, "Implementation and Performance Evaluation of the Gateway Discovery Approaches in the Integrated MANET Internet Scenerio", International Journal on Computer Science and Engineering, Vol.3 No.3, March 2011, PP: 1213-1226.

[7] Skloul Ibrahim, Dr. Peter J.B. King and Prof. Robert Pooley, "Performance Comparison of CBR in MDVZRP with DSDV and AODV".

[8] Kurose J.F, Ross K.W., "Computer Networking: A Top down Approach Featuring the Internet", 3rd edition, Addison Wesley (2003). 
[9] Network Working Group; "Routing Information Protoco"l; $\quad$ http://www.ietf.org/rfc/rfc1058.txt? umber $=1058$

[10] Network Working Group;" OSPF Version 2"; http://www.ietf.org/rfc/rfc1247.txt

[11] Network Working Group; "A Border Gateway Protocol (BGP)"; http://www.ietf.org/rfc/rfc1105.txt? Number $=1105$

[12] Kumar Rakesh,Anil Kumar Surje and Manoj Mishra "Review Strategies And Analysis of Mobile Ad hoc Networks-Internet Integration Solutions" IJCSI International Journal of Computer Science Issues, Vol. 7, Issue 4, No 6, July 2010

[13] C. E. Perkins, E. M. Royer, and S. R. Das, "IP Address Autoconfiguration for Ad Hoc Networks," Internet Draft, Nov. 2001, Work in progress.

[14] Droms R. "Dynamic host configuration protocol", IETF Network Working Group, RFC 2131

[15] Erik Nordstrom, Per Gunningberg and Christian Tschudin "Design of Internet Connectivity for Mobile Ad hoc Networks"

[16] Erik Nordström , Per Gunningberg and Christian Tschudin, "Robust and flexible Internet connectivity for mobile ad hoc networks",Elsevier- Ad Hoc Networks2011

[17] Kumar R. "Performance Evaluation of Gateway Discovery Approaches in the Integrated Mobile Ad Hoc Network (MANET)-Internet Scenario" International Journal of Computer Technology and Electronics Engineering (IJCTEE) Volume 2, Issue 3, June 2012

[18] Belding-Royer E.M., Sun Y., Perkins C.,"Global Connectivity for IPv4 Mobile Ad-Hoc Networks", IETF Internet Draft, Nov. 2001. Work in progress.

[19] Shiv Mehra and Chansu Yu. "Survey on Techniques Providing Internet Connectivity to Mobile Ad hoc Networks"

[20] U. Jonsson, F. Alriksson, T. Larsson, P. Johnasson, G.Q. Maguire," MIPMANET: Mobile IP for Mobile Ad-hoc Network"s, in: Proceedings of IEEE/ACM Workshop on Mobile and Ad Hoc Networking and Computing (MobiHoc'00), Boston, MA, USA, August 2000, pp. 7585.

[21] P. Ratanchandani and R. Kravets, "A Hybrid Approach to Internet Connectivity for Mobile Ad hoc Networks", in Proc. of the IEEE WCNC 2003, Vol. 3, pp. 15221527. New Orleans, USA, March 2003.

[22] Jaewook Shin, Haeryong Lee, Jeehyeon Na, Aesoon Park and Sangha Kim" Gateway Discovery and Routing in Ad Hoc Networks withNAT-based Internet Connectivity" 0-7803-8521-7/04/\$20.00 @ 2004 IEEE

[23] P. M. Ruiz and A. F. Gomez-Skarmeta, "Enhanced Internet Connectivity for Hybrid Ad Hoc Networks Through Adaptive Gateway Discovery", in Proc. of the 29th IEEE International Conference on Local Computer Networks (LCN'04), Tampa, Florida, November 2004

[24] P. M. Ruiz and A. F. Gomez Skarmeta, "Maximal Source Coverage Adaptive Gateway Discovery for Hybrid Ad
Hoc Networks", Lecture Notes in Computer Science, vol.3158, July 2004, pp.28-41.

[25] P. M. Ruiz and A. F. Gomez-Skarmeta, "Adaptive Gateway Discovery Mechanisms to Enhance Internet Connectivity for Mobile Ad Hoc Networks", Ad Hoc \& Sensor Wireless Networks, Vol. 1, 2005, pp. 159-177.

[26] Khaleel Ur Rahman Khan, Ehthesham M., Kumar M., Rafi UZaman, "An Effective Gateway Discovery Mechanism in an Integrated Internet-MANET (IIM)", IEEE, 2008.

[27] S. Bin, S. Bingxin, L. Bo, H. Zhonggong, and Z. Li, "Adaptive gateway Discovery Scheme for Connecting Mobile Ad Hoc Networks to the Internet," Proceedings of International Conference on Wireless Communications, Networking and Mobile Computing, vol. 2, pp. 795-799, 2005

[28] A. Hamidian, U. Korner, A. Nilsson, "Performance of internet access solution in mobile ad hoc networks", Lecture Notes in Computer Science 3427 (1) (2005) 189-201.

[29] R. Wakikawa, J.T. Maline, C.E. Perkins, A. Nilsson, A.H. Tuominen, "Global Connectivity for IPv6 Mobile Ad-hoc Networks", IEFT Internet-Draft, draftwakikawamanet-globalv6-05.txt, March 2006.

[30] Kumar Rakesh, and Misra, M. "An Efficient Mechanism for Connecting MANET and Internet through Complete Adaptive Gateway Discovery", In Proceedings of the First International Conference on Communication System Software and Middleware (COMSWARE2006). New Delhi, India, January 2006, 1-5.

[31] Yuste, A.J., Trujillo, F.D., Trivĩno, A., Casilari, E.: "An adaptive gateway discovery for mobile ad hoc networks". In: 5th ACM international workshop on Mobility management and wireless access (MOBIWAC), Chania, Crete Island, Greece, October 2007, pp. 159-162 (2007).

[32] Yuste, A.J., Trujillo, F.D., Trivĩno, A. and Casilari, E" Connectivity Gateway Discovery in MANETs" SpringerVerlag Berlin Heidelberg L. Cerd`a-Alabern (Ed.): Wireless and Mobility, LNCS 5122, pp. 128-141, 2008.

[33] Mari Carmen Domingo and Rui Prior " An Adaptive Gateway Discovery Algorithm to support QoS when providing Internet Access to Mobile Ad Hoc Networks" JOURNAL OF NETWORKS, VOL. 2, NO. 2, APRIL 2007.

[34] Francisco J. Ros, Pedro M. Ruiz "Low Overhead and Scalable Proxied Adaptive Gateway Discovery for Mobile Ad Hoc Networks Dept. of Information and Communications Engineering University of Murcia", Mobile Adhoc and Sensor Systems (MASS), 2008 IEEE InternationalConferenceon IssueDate:Oct.2008 On page(s):226 -235

[35] M. Geetha, Dr. R. Umarani, R. Kiruthika, "A Comparative Study of Gateway Discovery Protocol in MANET", International Journal of Computer Application, Vol.11 No.2, Dec.209, PP: 0875-555, December 2010.

[36] ZHUANG Lin, LIU Yuan-an, LIU Kai-ming, ZHAI Linbo and YANG Ming "An adaptive algorithm for connecting mobile ad hocnetwork to Internet with unidirectional links supported"Elsevier journal of The 
Journal of China Universities of Posts and Telecommunications-2010

[37] Shahid Md. Asif Iqball and Md. Humayun Kabir "HYBRID SCHEME FOR DISCOVERING AND SELECTING INTERNET GATEWAY IN MOBILE AD HOC NETWORK" International Journal of Wireless \& Mobile Networks (IJWMN) Vol. 3, No. 4, August 2011

[38] H. Ammari and H. El-Rewini," Integration of Mobile Ad Hoc Networks and the Internet using Mobile Gateways," Proceeding of the 4th International Workshop on Algorithms for Wireless, Mobile, Ad Hoc and Sensor Networks (WMAN04), Santa Fee, New Mexico, USA, April 26-30, 2004
[39] B. A. Kock and J. R. Schmidt, "Dynamic Mobile IP Routers in Ad Hoc Networks," IWWAN 2004, Int'1. Wksp. Wireless Ad-hoc Networks, OULU FINLAND, June 2004.

[40] Khaleel Ur Rahman Khan, A V Reddy and Rafi U Zaman, "An Efficient Integrated Routing Protocol for Interconnecting Mobile Ad Hoc Network and the Internet," International Journal of Computer and Electrical Engineering, vol. 1, No. 1, pp. 32-39, April 2009. 